\title{
Concept for Image Intensifier with CMOS Imager Output Interface
}

\author{
Paul J. Thomas ${ }^{2}$, Robert S. Allison ${ }^{1}$, Richard Hornsey ${ }^{1}$, Winnifred Wong ${ }^{1}$ \\ ${ }^{1}$ York University, Department of Computer Science, Toronto, Canada \\ ${ }^{2}$ Topaz Technology Inc., Toronto, Canada
}

\begin{abstract}
A concept is described for the detection and location of transient objects, in which a "pixel-binary" CMOS imager is used to give a very high effective frame rate for the imager. The sensitivity to incoming photons is enhanced by the use of an image intensifier in front of the imager. For faint signals and a high enough frame rate, a single "image" typically contains only a few photon or noise events. Only the event locations need be stored, rather than the full image. The processing of many such "fast frames" allows a composite image to be created. In the composite image, isolated noise events can be removed, photon shot noise effects can be spatially smoothed and moving objects can be de-blurred and assigned a velocity vector. Expected objects can be masked or removed by differencing methods. In this work, the concept of a combined image intensifier/CMOS imager is modeled. Sensitivity, location precision and other performance factors are assessed. Benchmark measurements are used to validate aspects of the model. Options for a custom CMOS imager design concept are identified within the context of the benefits and drawbacks of commercially available night vision devices and CMOS imagers.
\end{abstract}

KeyWords: Night Vision Goggles, CMOS imager, modeling

\section{INTRODUCTION}

When incorporated into a Night Vision Goggles (NVG), microchannel plate image intensifiers provide single-photon detection capability for the human eye, with a response time that is limited by the decay time constant of the phosphor screen and can approach $1 \mathrm{kHz}^{1,2,3}$. Ground-based and satellite sensor networks to monitor such objects are of practical interest. In the sky scene, stars and planets are quasi-stationary or move with a small angular velocity that is caused primarily by Earth rotation. The objects of interest are satellites in near-earth orbit, asteroids or similar transient objects passing close to Earth ${ }^{4}$. Such objects generally have an angular motion that is orders of magnitude larger than the stars. Most of the scene objects of interest are "faint" point sources. (Here, "faint" is taken to mean a signal of low signal to noise ratio (SNR) but still detectable and much lower than the detector saturation level.) The following discussion is restricted to such objects.

The conventional detection method is to use a telescope with a large aperture and small focal ratio, followed by a sensitive CCD array detector. The large aperture of the telescope maximizes the number of photons collected from a point source, while the small focal ratio increases the irradiance on the focal plane (fewer pixels in the point spread function). A sensitive CCD is needed, preferably cooled to reduce noise and backside-illuminated to increase the quantum efficiency ${ }^{5}$. In this scenario, the desired integration time is often seconds to minutes, which is a problem for moving objects like satellites.

\subsection{Effect of Source Motion}

For an array detector, source motion during the integration time can spread the signal electrons across multiple pixels, as depicted schematically in Figure 1(a). For each pixel, the noise is generally similar from pixel to pixel and adds to the output of the pixel regardless of the presence of a signal. On a specific pixel, the signal lasts for a time, $t_{d w e l l}$, that may depend on the apparent angular size of the object and is inversely proportional to its angular velocity across the image. In the present context, the upper limit to $t_{d w e l l}$ is $t_{\text {int }}$, the integration time. Throughout the integration time, noise electrons accumulate on each pixel at a constant rate. If the integration time of the detector is longer than the dwell time of the signal on a specific pixel, then the pixel receives added noise without any added signal, and the signal to noise ratio (SNR) is reduced. Figure 1(b) illustrates this concept with simulated data. The abscissa in the figure is the time since reset, expressed as a fraction of the integration time. Three different dwell times are shown. When $t_{d w e l l}=t_{\text {int }}$, the angular 
velocity is effectively zero and the maximum SNR is achieved. In the figure, the SNR varies due to random noise. In the other two curves of Figure 1(b), the velocity is not negligible and the dwell times are as shown. The SNR is the same for all traces until the signal ends, then is reduced by the added noise that arrives after the signal leaves the pixel. (In the figure, multi-pixel contributions, $\sqrt{\mathrm{N}}$ benefits of averaging and other effects have been ignored for clarity, but the basic conclusion of severely reduced SNR is not sensitive to these details.)
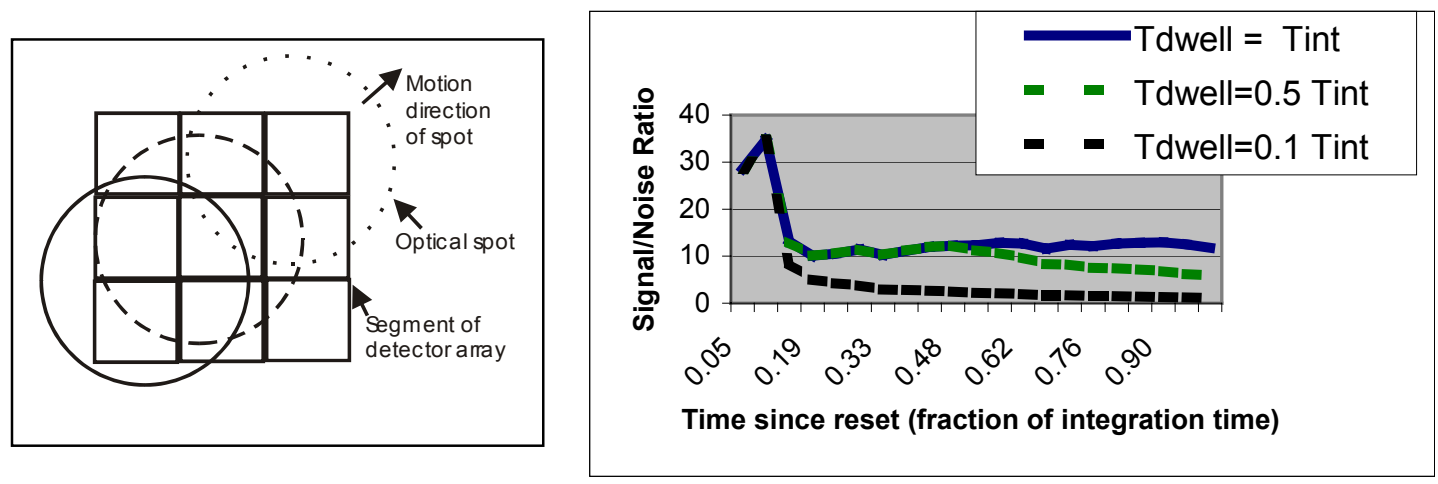

Figure 1 Source Motion During Integration (a) concept, (b) SNR Simulation

When the signal electrons are subsequently combined by averaging or other means, the noise is then proportional to the square root of the number of pixels being used in the averaging. Hence, it is preferable in low-signal situations (where the SNR limits the system performance) to concentrate all of the signal onto the smallest number of pixels. This statement is true when the detector noise is mainly from the noise of the dark current or from other sources that are proportional to the integration time. In some situations, the readout noise dominates and the above line of reasoning does not apply.

While it is possible to compensate for source apparent motion in the case of stars, as is often implemented with equatorial-mounted telescopes, it is difficult to do so for satellites or asteroids, especially if such events occur in an unpredictable manner and with velocity vectors that may not be known a-priori. Hence, it is often desirable to match the integration time of the signal on a detector array to the angular motion of the source, so that integration of noise on a pixel stops once the signal no longer falls on that pixel. Longer effective integration times can then be achieved by combining images with a translation between images such that the signals are additive. This strategy minimizes the number of pixels whose noise contributes to SNR reduction.

\subsection{Requirement of Integration Time Matched to Source Velocity}

In order to match the integration time of the sensor to the angular velocity of a sky source, a variety of factors that merit consideration are listed in Table 1.

\section{Table 1 Factors in Compensation for Source Velocity}

\begin{tabular}{|l|l|}
\hline \multicolumn{1}{|c|}{ Factor for Consideration } & \multicolumn{1}{c|}{ Notes } \\
\hline $\begin{array}{l}\text { "Angular resolution" of system } \\
\text { (centroid-determination precision) }\end{array}$ & $\begin{array}{l}\text { Angular resolution depends on the telescope focal length and the centroid- } \\
\text { determination capability of the detector and its associated processing } \\
\text { electronics. }\end{array}$ \\
\hline Update rate & $\begin{array}{l}\text { The detector frame rate must be faster than the transit time of the image } \\
\text { through a pixel, and the image processing must be able to keep pace with the } \\
\text { frame rate. }\end{array}$ \\
\hline Sensitivity and noise & $\begin{array}{l}\text { Sky sensors must be able to tolerate direct views of the moon, planets or bright } \\
\text { stars, albeit for brief durations. }\end{array}$ \\
\hline Saturation and sensor damage & $\begin{array}{l}\text { In many sky-viewing situations, a large total field is desirable without } \\
\text { compromising angular resolution. }\end{array}$ \\
\hline Total field of view &
\end{tabular}


These factors are clarified in the context of a novel instrument that uses a telescope/image intensifier front end to bring signals to a custom CMOS imager. The output of the imager is "event locations" that are used to determine object positions and velocities and to compensate for object motion. The term, "angular resolution", for a spot is used here to mean the precision in the location of an object in the sky, which is equivalent to the determination of the spot centroid in the corresponding image. This "angular resolution" is not related to the Rayleigh or similar resolution criteria that are intended to resolve nearby objects ${ }^{3}$. The centroid precision and accuracy are not directly affected by the "blur size" (point spread function) of the image of the sky source on the detector ${ }^{6}$. Rather, it is the ability to determine the centroid of the image spot that is important. The focal length of the telescope lens sets the scale factor for angular resolution. Drift and calibration issues are ignored in the present discussion.

A fast frame rate of the detector array is an essential prerequisite for the matching of integration time to the source velocity of a scene object. The architecture of the "pixel-binary" CMOS imager chip ${ }^{7,8}$ is well-suited to the 'parallel' readout of centroid information in a time that is limited by the response time of the on-pixel comparators. A serial readout of analog pixel data is not needed in this architecture. When a conventional CMOS imager is used for the present application, the effective frame rate can be increased to $100 \mathrm{~Hz}$ or more by reading out only a region of interest (ROI) around the image to be analyzed. This method requires differencing or other preprocessing to identify objects of interest and thereby define the region(s) of interest. In the "pixel-binary" imager, regions of interest can also be of value for noise suppression.

Whereas a conventional CCD imager generally needs $10^{2}-10^{3}$ photoelectrons to identify the presence of an object in the image $^{5,6}$, an image intensifier can achieve the same effect with $10^{1}$ photoelectrons because of its low noise rate.

Furthermore, the GaAs photocathode ${ }^{9,10}$ of a "Gen III" image intensifier has a spectral distribution of responsivity that is better matched to the solar spectrum than most solid state imagers. (Both asteroids and satellites are generally detected by means of the diffuse or specular reflection of sunlight.) The noise in an NVG, when used in the present application, can be reduced to negligible proportions by spatial and temporal filtering and thresholds.

Saturation can be a limiting factor for the use of NVG, even though they routinely operate over a dynamic range of $10^{6}$ or more. This dynamic range is achieved by automatic gain control (AGC) circuitry that reduces the NVG gain when the average scene intensity is high. Both short-term inoperability (eg high noise) and long-term damage (to the photocathode through ablation and chemical changes) are possible. In the present work, saturation/damage effects are ignored. It is assumed that an external shutter mechanism can prevent catastrophic damage. Likewise, the total field of view of the sensor system is not considered here.

In Section 2 below, the instrument concept and the major subsystems are described in more detail. Section 3 analyzes the system to determine the expected performance in selected areas. A software model is outlined in Section 4. The potential of a custom NVG and CMOS imager for the present application are assessed in Section 5.

\section{DESCRIPTION OF NEW INSTRUMENT CONCEPT}

In the instrument concept described here, the objective lens of a commercial Night Vision Goggles (NVG) is replaced by a small telescope. At the output of the NVG, the eyepiece is replaced by coupling optics that image the fluorescent screen of the NVG onto the focal plane of a CMOS imager. Additional computing or other circuitry can be used to provide an output of 'object locations' rather than imagery. Figure 2 summarizes the concept. High sensitivity and high equivalent frame rate are expected for this instrument concept. The end result is a system that, under suitable illumination conditions, can track a rapidly moving transient object while providing singlephoton sensitivity, wide dynamic range and good angular location accuracy. The key features of the instrument concept are:

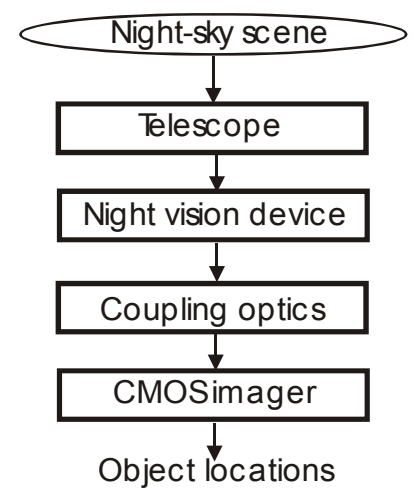

Figure 2 Instrument Concept: CMOS Imager with NVG Front End 
- Ability of the CMOS imager to detect the centroid of the 'spot' created by the cluster of photons emitted by the NVG when a single photocathode event occurs;

- Ability of the CMOS imager and its post processing to cluster the 'spot' centroids from individual photoevents to create the image of a scene object with subpixel precision;

- Fast response time of the NVG (circa $10^{3} \mathrm{~Hz}$ for off-the-shelf devices); note that a vastly improved NVG response time is possible with a custom device;

- The fast imager response possible with custom 'pixel-binary' CMOS imagers and on-chip centroid calculation;

- Coupling optics that efficiently transfer the fluorescent output of the NVG to the imager active surface.

The principal subsystems, per Figure 2, are the telescope, the NVG, the coupling optics and the CMOS imager. Each subsystem is described below, including the inputs, the outputs and other parameters used for performance modeling.

\subsection{Telescope}

A simple-lens telescope model is used ${ }^{11}$, with parameters as listed in Table 2. Note that the actual optical component can be multi-element or reflective to improve the specifications. The default values in the table are for an off-the-shelf $\mathrm{C}$-mount lens. For convenience in simulations, the optic axis of the telescope is assumed to be the z-axis of the system coordinate system and the telescope is assumed to be rotationally symmetrical. The field of view and blur spot size are determined by measurement. Stray light and scattered light are ignored in this application, and the objects of interest are assumed to be at infinity. The system concept is not sensitive to any of the telescope parameter values. The input to the telescope is the irradiance from the scene, while the telescope output irradiance distribution is imaged to fall on the (photo-)active area of the detector array.

Table 2 Parameters of a Representative Telescope

\begin{tabular}{|l|c|c|l|}
\hline \multicolumn{1}{|c|}{ Parameter } & units & $\begin{array}{c}\text { Nominal } \\
\text { value }\end{array}$ & \multicolumn{1}{|c|}{ Notes } \\
\hline Aperture & $\mathrm{mm}$ & 40 & Commercial C-mount lens \\
\hline Focal length & $\mathrm{mm}$ & 75 & $\begin{array}{l}\text { Selected for adequate sky performance and convenient } \\
\text { operation in the laboratory }\end{array}$ \\
\hline Focal ratio & - & 1.9 & Ratio of focal length over aperture. \\
\hline Theta_opt_axis & Degrees & 0 & attitude angle of orientation of telescope axis \\
\hline Phi_opt_axis & Degrees & 0 & Azimuth angle of orientation of telescope axis \\
\hline Theta_fov & $\mathrm{sr}$ & variable & $\begin{array}{l}\text { Field of view solid angle of telescope; may also be } \\
\text { limited by the dimensions of the detector array. } \\
\text { Determined by measurement }\end{array}$ \\
\hline Image_distance & $\mathrm{mm}$ & Focal length & For sky objects at infinity. \\
\hline Iscatt & $\mathrm{W} / \mathrm{cm}^{2}$ & 0 & Light scattered by the optical path \\
\hline Istray & $\mathrm{W} / \mathrm{cm}^{2}$ & 0 & $\begin{array}{l}\text { Light reflected in unwanted manner by telescope or } \\
\text { other instrument components. }\end{array}$ \\
\hline Throughput & - & 0.8 & Estimated average over spectral region of NVG \\
\hline Blur_spot & $\mathrm{um}$ & $(30)$ & Determined by measurement \\
\hline Aberrations & - & - & Ignored in initial model. \\
\hline
\end{tabular}

\subsection{NVG}

The input to the night vision goggles is a low-intensity optical irradiance distribution, while the output is a spatially faithful representation of the input where each input photoelectron has been replaced by a large number, "gain" of photons. Single-photoelectron events can be readily detected. Shot noise, saturation and other effects are not included in this brief overview. A representative NVG (night vision goggles) is shown schematically in Figure 3. Mechanical and electrical details that are not needed in the present discussion are omitted. During operation, the irradiance distribution from the telescope falls on the photocathode plane of the NVG. The electrons created by the photocathode (in a Poisson 
random process) in response to the irradiance are incident on the microchannel plate and multiplied by the gain of the NVG while perhaps losing some resolution due to electron spreading and the discreteness of the microchannel plate and the fiber image guide. A fluorescent screen in close proximity to the output of the microchannel plate converts the electrons back to photons. Each electron emitted by the photocathode provides a large burst of electrons at the fluorescent screen within a short time period, typically a few nanoseconds. Electrostatic focusing can be used to reduce the angular spread of the electrons. Whereas an eyepiece and a human observer complete the NVG model, coupling optics and a CMOS imager capture the fluorescent emission in the present scenario. Typically, the output of the fluorescent screen of an NVG is coupled into a fiber image guide, to simplify the design of the eyepiece optics.

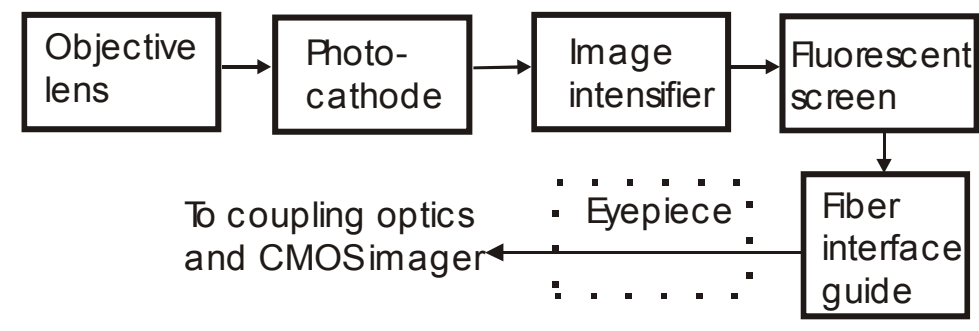

Figure 3 Night Vision Goggles Block Diagram

A microchannel plate (MCP) consists of a glass plate with a dense array of holes of uniform spacing and diameter. A typical hole ('pore' or 'channel') might have a cross-sectional diameter of $12 \mu \mathrm{m}$ or less. A bias voltage is applied between the surfaces of the plate. Electrons that hit the inner wall of a channel cause the emission of additional electrons that are, in turn, accelerated down the channel. A bias voltage of about $1-2 \mathrm{kV}$ is generally sufficient to give a gain that can vary from $10^{3}$ to $10^{6}$ or more depending on the design. Multiple plates can be stacked for enhanced noise rejection. The MCP bias voltage in an NVG is typically controlled by the average scene illumination in order to provide automatic gain control and enhance the dynamic range. For instance, the average current to the MCP can have an upper limit that is maintained by reducing the bias voltage. Representative parameters of the NVG are listed in Table 3. Note that noise sources other than signal shot noise are ignored to first order, as is the spatial variation of the gain.

Table 3 Parameters of Typical NVG

\begin{tabular}{|l|c|c|l|}
\hline \multicolumn{1}{|c|}{ Parameter } & units & $\begin{array}{c}\text { Nominal } \\
\text { value }\end{array}$ & \multicolumn{1}{|c|}{ Notes } \\
\hline Quantum efficiency & - & 0.2 & GaAs photocathode ${ }^{9,10}$ \\
\hline Gain & - & $10^{6}$ & Performance is not sensitive to gain \\
\hline Fluorescent efficiency & - & 1 & \\
\hline Fluorescence lifetime & $\mathrm{msec}$ & 0.7 & For a P43 photocathode ${ }^{11}$ \\
\hline $\begin{array}{l}\text { Fluorescence angular } \\
\text { distribution }\end{array}$ & - & lambertian & $\begin{array}{l}\text { Equal emission in all directions, but including } \\
\text { the effects of the optical window. }\end{array}$ \\
\hline Gain spatial variation & - & 0 & Spatial variation is ignored, to first order. \\
\hline Gain voltage noise & Events/pore/second & 0 & $\begin{array}{l}\text { Other than signal shot noise, noise sources are } \\
\text { ignored, to first order }\end{array}$ \\
\hline Positive ion noise & Events/pore/second & 0 & \\
\hline Pore noise & Events/pore/second & 0 & $\begin{array}{l}\text { Assumes AGC fixed at maximum gain (ie, } \\
\text { bright spot in otherwise dark scene) }\end{array}$ \\
\hline Saturation level & $\begin{array}{c}\text { Signal for which } \\
\text { SNR=1 }\end{array}$ & 100 & Ignored here \\
\hline Halo size parameter & & tbd & \\
\hline
\end{tabular}

The fluorescence efficiency is set to unity. For the present application, the operation of the NVG is insensitive to most parameter variations that can reasonably be expected to occur. An interesting parameter of the NVG is the saturation level, representing the "maximum signal". This term is constrained to the situation where a high-intensity, short optical pulse is applied from a point source, so that the NVG sees a short-duration (compared to the fluorescence lifetime of the phosphor) optical irradiance that is concentrated on a spot comparable in order of magnitude to the blur spot of the NVG. 
These conditions are defined to exclude operation of the AGC of the NVG. It is expected that the gain of the MCP drops dramatically during such a pulse of illumination, due to the draining of the charge along the pores. The recharging time of the MCP affects this definition as well. An NVG has the potential for localizing the effect of saturation. For the present work saturation effects will not be considered further.

\subsection{CMOS Imager}

The CMOS imager described here (Table 4) is a commercial unit used for initial evaluations of the concept. The quantum efficiency is a nominal value. Tentative specifications for a custom "pixel-binary" CMOS imager are also included. The effective frame rate is expected to be greater than $10^{4} \mathrm{~Hz}$, while the quantum efficiency is reduced by the smaller fill factor (occupied by on-pixel comparators and digital circuitry). As feature sizes shrink, the thickness of the photosensitive layer and other factors tend to reduce the quantum efficiency in imagers fabricated with standard CMOS processing $^{7}$.

Table 4 Parameters of CMOS Imager

\begin{tabular}{|c|c|c|c|c|}
\hline Parameter & units & $\begin{array}{l}\text { Nominal } \\
\text { Value } \\
\text { (off-shelf) }\end{array}$ & $\begin{array}{c}\text { Nominal } \\
\text { Value } \\
\text { (Pixel-binary) }\end{array}$ & Notes \\
\hline $\begin{array}{l}\text { Quantum } \\
\text { efficiency }\end{array}$ & $\begin{array}{l}\text { Electrons/ } \\
\text { photon }\end{array}$ & 0.2 & $(0.05)$ & $\begin{array}{l}\text { Value is nominal and averaged for bandpass. Affected } \\
\text { by wavelength and pixel fill factor. }\end{array}$ \\
\hline Pixel dimension & um & 6 & 15 & Square pixel \\
\hline Number of rows & - & 640 & 128 & Can be reduced using a region-of-interest readout \\
\hline $\begin{array}{l}\text { Number of } \\
\text { columns }\end{array}$ & - & 480 & 128 & Can be reduced using a region-of-interest readout \\
\hline $\begin{array}{l}\text { Equivalent } \\
\text { Frame rate }\end{array}$ & $\mathrm{Hz}$ & $10^{2}$ & $10^{5}$ & $\begin{array}{l}\text { Depends on the size of the region of interest, the type of } \\
\text { imager and other factors. Pixel-binary method reads } \\
\text { out the centroid directly. }\end{array}$ \\
\hline $\begin{array}{l}\text { Spatial response } \\
\text { nonuniformity }\end{array}$ & $\%$ & - & - & Ignored here \\
\hline Bad pixels & - & - & - & $\begin{array}{l}\text { Ignored here; optical spot covers many pixels in most } \\
\text { cases. }\end{array}$ \\
\hline $\begin{array}{l}\text { Saturation and } \\
\text { dynamic range }\end{array}$ & - & - & - & $\begin{array}{l}\text { Ignored here; high effective frame rate of pixel-binary } \\
\text { usually precludes saturation effects. }\end{array}$ \\
\hline Blooming & - & - & - & Ignored here; depends on reset strategy \\
\hline
\end{tabular}

The CMOS imager needs to be able to detect the NVG signal burst that is associated with a photocathode event. Furthermore, the imager and follow-on electronics should allow such a signal to be read out and processed before the next photocathode event occurs. This last criterion severely limits the maximum irradiance that can be handled. The dynamic range of the irradiance is improved by a higher frame rate. The "pixel-binary" custom CMOS imager ${ }^{7,8}$ is expected to allow a centroid rate in excess of $10^{5} \mathrm{~Hz}$. "Region of interest" readout schemes can also facilitate a larger dynamic range. It is also important to mask off bright scene objects and, in fact, any scene objects (eg stars) that are not of interest.

\subsection{Coupling Optics}

The concept for the coupling optics is shown in Figure 4. A key design variable is the spot size on the image plane of the detector array. Although the centroiding algorithm may work better with larger spot diameters, the number of photons per pixel is reduced. In the present situation, the number of photons at the detector per photocathode event, while large, is still expected to be a limiting factor in the performance. Hence, the design should minimize the spacing between the image guide and the "pixel plane" of the detector array and the spacing between the fluorescent plane and the input to the image guide. The instrument concept is also viable with a coupling lens (or lens array) between the image guide and the detector array. In this way, the spot dimension on the detector array can be adjusted by the lens/lens 


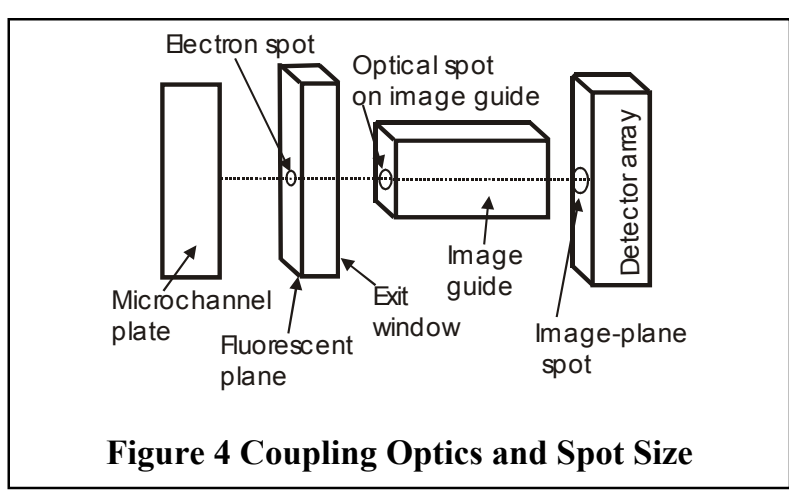

array independent of the spot dimensions coming into the image guide. Additional package size and mass are introduced by the lens, however, and the photon throughput can be reduced.

\section{ANALYSIS OF SYSTEM PERFORMANCE}

Table 5 shows some important system performance factors for the satellite tracking application. The intent of these analyses is to demonstrate the ability of available components to implement the instrument concept for the satellite-location application, and to identify areas where changes to the component specifications can significantly improve the system performance.

Sensitivity is of primary importance. The CMOS imager must be able to detect a photocathode event. Because events are the basic detection item, it is more appropriate to address noise in terms of probability of detection $\left(\mathrm{P}_{\mathrm{d}}\right)$ and false alarm rate $(\mathrm{FAR})^{5,13}$ rather than detector or NVG noise parameters. In order to distinguish successive photocathode events, a fast update rate for the imager is essential. "Fast" is normalized to the decay halfwidth of the fluorescent screen of the NVG, which sets the approximate duration of a photocathode event. This temporal normalization also determines the maximum angular velocity of the object before the signal spreads across multiple pixels. To obtain a useful centroid location, the post-processing should be able to keep pace with the event rate. This factor may limit dynamic range, determine the region of interest strategy and militate the masking of bright background objects. Finally, the coupling optics are important and are evaluated for their impact on the system performance factors.

\section{Table 5 Important System Performance Factors for Satellite Location}

\begin{tabular}{|l|l|}
\hline \multicolumn{1}{|c|}{ Feature } & \multicolumn{1}{c|}{ Notes } \\
\hline $\begin{array}{l}\text { Sensitivity and noise } \\
\text { rejection }\end{array}$ & $\begin{array}{l}\text { SNR of detection of a photocathode event, and probability of a noise } \\
\text { event. }\end{array}$ \\
\hline Temporal response & $\begin{array}{l}\text { Currently-available NVG devices have a temporal response that is } \\
\text { governed by the decay time of the fluorescent screen (700 } \mu \text { sec for } \\
\text { P43 material }{ }^{12} .\end{array}$ \\
\hline $\begin{array}{l}\text { Centroid location and } \\
\text { resolution }\end{array}$ & Can achieve +-0.1 pixel or better \\
\hline Background objects & $\begin{array}{l}\text { Masking, differencing and other hardware and software image } \\
\text { processing methods are available. }\end{array}$ \\
\hline Large signals & For convenience, these effects are ignored to first order. \\
\hline Coupling optics & $\begin{array}{l}\text { Affects sensitivity. Mass, size, stray light are deemed to be secondary } \\
\text { factors and are not considered here. }\end{array}$ \\
\hline
\end{tabular}

\subsection{Sensitivity and Noise Rejection}

Sensitivity is determined by the quantum efficiency of the photocathode of the NVG over the spectral region of the sky objects and considering the transmission of the atmosphere and the reflectivity of the satellite. Sunlight can be modeled by a blackbody with a temperature of about $5400 \mathrm{~K}$ and the atmospheric transmission is well known ${ }^{5}$. For the purposes of this work, the spectral reflectance of the target object (eg satellite) is assumed to be uniform over the spectral region of interest. For such a source, the effective target radiance increases throughout the visible and near infrared spectral regions.

The gallium arsenide photocathode of the "Gen III" NVG has a broad region of high quantum efficiency that extends from about 400 to $900 \mathrm{~nm}^{9,10}$. By contrast, many CCD imagers have reduced spectral response beyond perhaps 700 to $800 \mathrm{~nm}^{5}$, a region where the solar spectrum is intense ${ }^{11}$. 
The expected single-photoelectron signal per pixel follows Equation (1).

$$
N_{s i g} \cong \frac{G_{f l} G_{i i} \Omega_{\text {guide }}}{\left[\frac{\pi}{4} \frac{d^{2} \text { blur }}{d^{2}{ }_{p i x}}\right]} \tau_{o p t} Q E_{i m}
$$

Here, nominal values of the fluorescence gain, $\mathrm{G}_{\mathrm{fl}}$, the image intensifier gain, $\mathrm{G}_{\mathrm{ii}}$, the image guide collection solid angle, $\Omega_{\text {guide }}$ (for light from the fluorescent screen), and the blur spot dimension on the imager, $\mathrm{d}_{\text {blur }}$ are found in Tables 3 and $\mathbf{4}$. Note that the blur spot is approximately expressed in terms of pixels by dividing by the pixel area, $\mathrm{d}_{\text {pix. }}^{2}$ The throughput of the optics is $t_{\text {opt }}$ and the quantum efficiency of the imager is $\mathrm{QE}_{\mathrm{im}}$. Preliminary simulation results using Equation (1) are shown in Figure 5.

The low-noise feature of the NVG is a key factor in this application, and is described further in Section 3.2 below.

\subsection{Temporal Response}

The gain of the NVG produces a burst of photons at the fluorescent screen. This cluster of photons is generated within a couple of decay halfwidths for the NVG (Figure 6) and has limited spatial extent (ie, forms a brief 'spot'). Temporal response

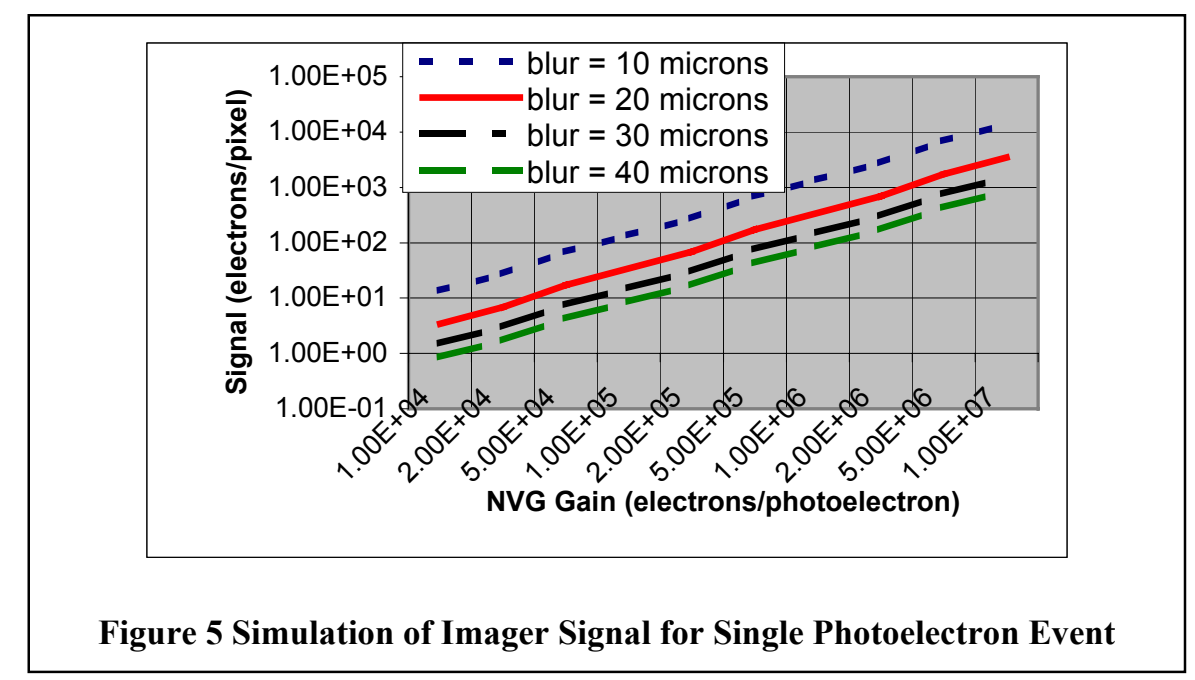
has several factors: photocathode and image intensifier lag, decay time of fluorescence of phosphor screen of NVG, scattered light, readout time of imager and analysis time. Photocathode and image intensifier lag tend to be less than $10^{-8}$ $\mathrm{sec}^{9}$ and can be ignored. The temporal response of the NVG is dominated by the decay time of the phosphor screen. In Figure 6, the measurements ${ }^{13}$ are for a P43 phosphor $^{11}$.

In Figure 6(a), the variation of the halfwidth is shown. The background noise on the time trace (Figure 6(b)) is very small, and has been enhanced by a factor of about $10^{3}$ in the trace labeled "aperture $-3 \mathrm{~mm}$ ". These traces indicate that a simple threshold should eliminate virtually all NVG noise that does not originate at the photocathode.
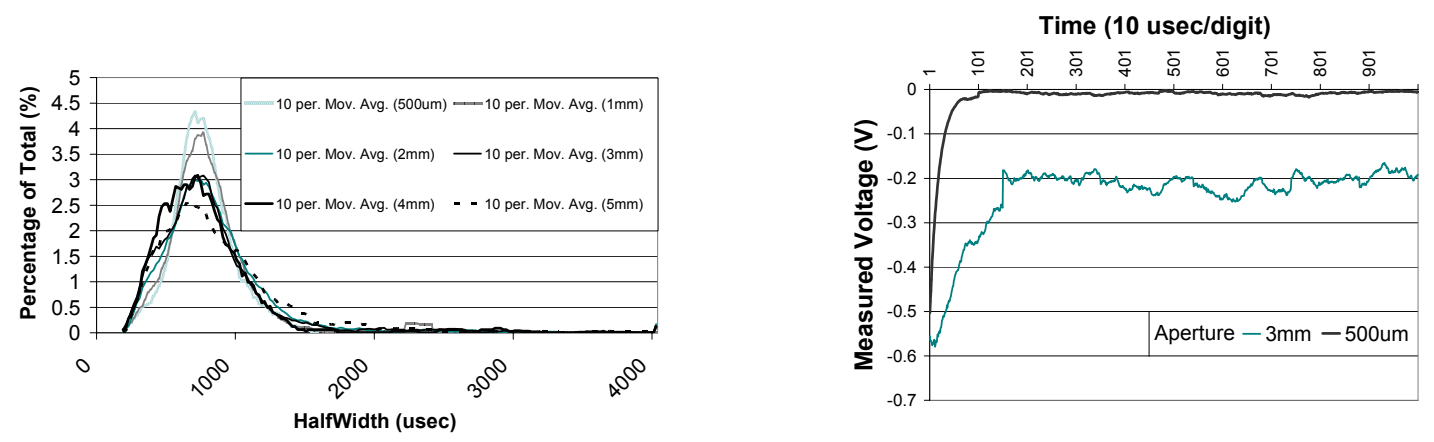

Figure 6 Fluorescence decay for Representative NVG: (a) histogram and (b) time traces 
The pixel-binary CMOS imager ${ }^{7,8}$ has a response time on the order of $10 \mu$ sec, which is $70 \mathrm{x}$ faster than the above decay time. Hence, such an imager can follow single photocathode events. By contrast, a representative commercial CMOS imager has a minimum frame rate of perhaps $10^{-2}$ seconds, which is an order of magnitude slower than is needed. Note that the frame rate of the imager rather than the integration time that is the important factor. Initial laboratory measurements used a commercial imager, and hence were limited in dynamic range by the CMOS imager.

\subsection{Centroid location and Resolution}

For the scenario described here, each photoelectron created at the photocathode of the NVG passes through its region of the image intensifier without strongly affecting other regions of the NVG. The centroid is unaffected by other events and the CMOS imager calculates the location immediately after the event occurs. Clusters of events (in time and location on the imager) are grouped to determine the location of the centroid of a 'scene event'. Such events also move in time. The fast response of the NVG allows the temporal motions to be clustered with additional modeling, to the extent allowed by limitations in signal strength. Preliminary evaluations of centroid detection indicate that the precision of the centroiddetermination is sensitive to the SNR of the pixel signals. A detailed estimation of the relationship between sensitivity and centroid precision is under investigation. Likewise, the errors introduced by the tracking mechanism for moving targets are currently being modeled.

\subsection{Background and Large Signals}

In a practical system, background stars, while sparse, will provide a significant signal count even when the image update rate is fast. Such signals can be masked. Among the various methods of masking, the use of "region-of-interest" masks on the detector array is promising. In this technique, slowly-varying objects, such as stars, can have a preset number of pixels blanked in a region defined by the centroid of the star. Occasional 'maintenance' images can be used to update the region-of-interest boundaries. In this way, most stationary signals can be masked. The "down-side" includes the time required for maintenance images and the fraction of the imager that is unavailable for sensing targets of interest. Based on the number density of stars of different stellar magnitudes, it is estimated that the maintenance time is less than $5 \%$ and that more than $90 \%$ of the image will be available for objects of interest. Since these objects are fast-moving, they will seldom be completely masked by the above method.

\subsection{Coupling Optics}

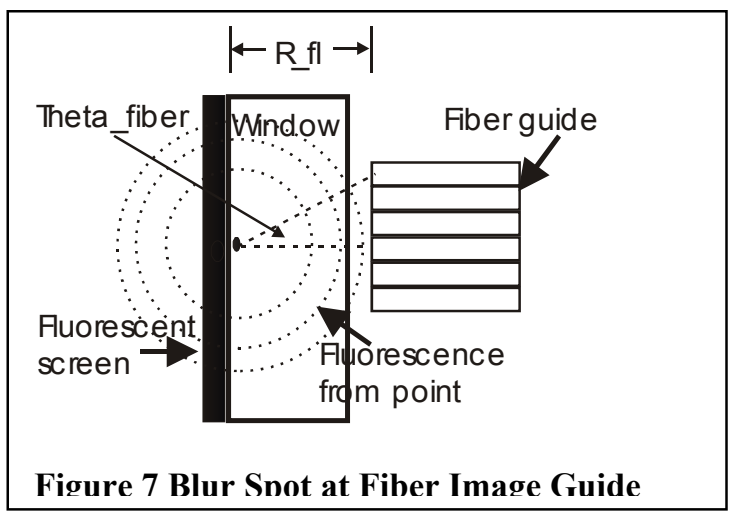

The key issue with the coupling optics is the increase of the blur spot size due to the spacings between the image guide and the fluorescent plane on the input side and the photo-active surface of the detector array on the output side. Figure 7 illustrates the spreading from the fluorescent screen. The fiber image guide is assumed to fill the field of view at a distance $r_{f l}$ from the screen. The corresponding angular size is given by the acceptance angle, $\theta_{\text {fiber }}$, of the individual fibers in the image guide. A typical number might be 55 degrees (numerical aperture of 0.53 ). The size of the spot on the input end of the fiber image guide is then $d_{\text {guide }} \cong d_{f l}+2 r_{f l} \tan \left(\theta_{f i b e r}\right)$. Even with zero spacing between the fiber guide and the window and a small spot, dfl, on the fluorescent screen, the thickness of the exit window increases the blur spot size. This spreading is minimized in a custom system in which the imager active surface replaces the window and fluorescent screen.

At the input side of the image guide, the spacing must be at least as large as the thickness of the exit window of the NVG, while on the output side, the constraint on the spacing is the bonding wires on the imager chip. It is assumed that the protective window on the imager package can be removed. In preliminary measurements, a C-mount lens was used to couple the output of the NVG into the CMOS imager. This method is inefficient in coupling NVG light into the imager. 


\section{IMPLEMENTATION OF THE SYSTEM MODEL}

A simple model of the NVG/imager system was implemented in Matlab, in order to assist in the design of a laboratory prototype incorporating a custom "pixel-binary" CMOS imager. Each subsystem of the model (Table 6) was separately coded. The NVG elements of the model have been reported previously ${ }^{6}$, while the theoretical foundation for the other elements of the model are well-documented in the literature ${ }^{12,13,14}$. Initial tests with the model verify the design concepts presented here, in general terms. Additional modeling is ongoing.

Table 6 Inputs and Outputs of the Subsystems of the Model

\begin{tabular}{|c|c|c|c|}
\hline element & inputs & outputs & Internal parameters \\
\hline Scene & $\begin{array}{l}\text { From user screen and a } \\
\text { set of test images of sky } \\
\text { scenes. }\end{array}$ & $\begin{array}{l}\text { Spectral radiance at } \\
\text { telescope, vs angle }\end{array}$ & $\begin{array}{l}\text { Location, angular size, radiance, } \\
\text { velocity, range, spectrum, variance, } \\
\text { other }\end{array}$ \\
\hline Telescope & $\begin{array}{l}\text { Spectral radiance at } \\
\text { telescope, vs angle }\end{array}$ & $\begin{array}{l}\text { Spectral Irradiance at } \\
\text { photocathode plane }\end{array}$ & $\begin{array}{l}\text { Throughput, blur spot, focal length, } \\
\text { aperture, obscuration, stray light, } \\
\text { scatter. }\end{array}$ \\
\hline $\begin{array}{l}\text { Image } \\
\text { intensifier }\end{array}$ & $\begin{array}{l}\text { Spectral Irradiance at } \\
\text { photocathode plane }\end{array}$ & $\begin{array}{l}\text { Fluorescence radiance } \\
\text { vs angle }\end{array}$ & $\begin{array}{l}\text { QE, gain, noise, halo effects, blur } \\
\text { spot. }\end{array}$ \\
\hline $\begin{array}{l}\text { Coupling } \\
\text { optics }\end{array}$ & $\begin{array}{l}\text { Fluorescence radiance } \\
\text { vs angle }\end{array}$ & $\begin{array}{l}\text { Irradiance at CMOS } \\
\text { imager surface }\end{array}$ & $\begin{array}{l}\text { Throughput, scatter, spreading, fiber } \\
\text { diameter, crosstalk, }\end{array}$ \\
\hline $\begin{array}{l}\text { CMOS } \\
\text { imager }\end{array}$ & $\begin{array}{l}\text { Irradiance at CMOS } \\
\text { imager surface }\end{array}$ & Analog signal by pixel & Slope, offset, noise, saturation, \\
\hline $\begin{array}{l}\text { Pre- } \\
\text { processing }\end{array}$ & Analog signal by pixel & $\begin{array}{l}\text { Event location, event } \\
\text { parameters }\end{array}$ & Threshold, bandwidth, other \\
\hline $\begin{array}{l}\text { Post- } \\
\text { processing }\end{array}$ & $\begin{array}{l}\text { Event location, event } \\
\text { parameters }\end{array}$ & Reconstructed image & $\begin{array}{l}\text { Averaging, anomaly removal, } \\
\text { nonuniformity correction. }\end{array}$ \\
\hline Display & Reconstructed image & Image on 'screen' & $\begin{array}{l}\text { Resolution, bandwidth, uniformity, } \\
\text { fidelity. }\end{array}$ \\
\hline
\end{tabular}

\section{CUSTOM COMPONENTS}

For the NVG, the most important factor is a reduction in the fluorescence decay time at the output stage. This change would allow the use of a faster imager update rate, a greater photocathode event rate and, consequently, a larger system dynamic range. Additional performance-enhancement factors for the NVG include higher gain and smaller point spread function. In order to circumvent the AGC features of a conventional NVG, it may be appropriate to control the NVG gain on a regional basis. A fundamental limitation of the proposed instrument is the total signal load on the NVG and the potential for permanent damage at bright spots in the image. Regional partitioning of the AGC will help to mitigate this problem while still allowing most of the NVG to operate at maximum gain.

Custom CMOS imager components ${ }^{15}$ have great flexibility in design, and can significantly enhance the performance of the instrument concept described here. The CMOS imager needs to have a response time that is matched to the fluorescence decay time of the NVG. In addition, the readout and processing of the imager information must keep pace with the response time in order to optimize the dynamic range of the system and allow noise reduction through on-chip and off-chip processing.

Table 7 identifies various options for features of a custom imager. Some of these options have been included in existing custom chips (Chips A, B and C), while Chip D, the custom rangefinder chip, is in the design phase at present.

Pixel-binary imagers designed for rangefinding ${ }^{8,16}$ can have an equivalent frame rate for the location of a centroid that is $10^{5} \mathrm{~Hz}$ or faster. This high rate is achieved by on-pixel comparators and row- (and column-) parallel readouts followed by suitable on-chip processing. In the pixel-binary scheme, selection of the threshold value for the comparators is a critical step. This has been done globally, but could benefit from local, adaptive thresholding. Another limitation of the 
pixel-binary method as currently applied is susceptibility to single-pixel noise ("outliers"). Various temporal and spatial methods for outlier removal are identified in the table.

Table 7 Options for Custom CMOS Imager Features

\begin{tabular}{|c|c|c|c|c|c|}
\hline $\begin{array}{l}\text { On-Chip } \\
\text { Feature }\end{array}$ & Chip & $\begin{array}{l}\text { Chip } \\
\text { B }\end{array}$ & Chip & $\begin{array}{l}\text { Chip } \\
\text { D }\end{array}$ & Notes \\
\hline $\begin{array}{l}\text { Real-time pixel } \\
\text { processing }\end{array}$ & YES & YES & YES & YES & $\begin{array}{l}\text { High frame processing rates achieved through pixel } \\
\text { binarization and/or row- (or column-) parallel readout. }\end{array}$ \\
\hline Centroid calculation & MTOPS & CCS & $\mathrm{AE}$ & YES & $\begin{array}{l}\text { MTOPS - Multi-Threshold Object Positioning System; } \\
\text { CCS - Cumulative Cross-section Summation; } \\
\text { AE - Accelerated Erosion. }\end{array}$ \\
\hline Pixel-level reset & NO & NO & NO & YES & $\begin{array}{l}\text { Ability to manually reset specific pixels. Exclusion of } \\
\text { bad pixels. }\end{array}$ \\
\hline Cluster manipulation & NO & NO & NO & YES & $\begin{array}{l}\text { Velocity/motion tracking of objects across frames; } \\
\text { noise reduction. }\end{array}$ \\
\hline Outlier removal & $\mathrm{NO}$ & YES & YES & YES & Noise suppression; ability to use a smaller threshold \\
\hline $\begin{array}{l}\text { Region of Interest } \\
\text { Operators }\end{array}$ & NO & YES & NO & YES & $\begin{array}{l}\text { Ability to read from, or mask off, specific windows of } \\
\text { interest; Adaptive local or region-of-interest threshold. }\end{array}$ \\
\hline
\end{tabular}

Other factors of value in a rangefinder system include a time stamp, the ability to perform a calibration transformation, power control and averaging. Although an on-chip solution is possible for these additional factors, some of them may also be readily implemented in programmable logic. The corresponding tradeoff includes cost and other criteria and is outside the scope of the present work.

\section{REFERENCES}

1. G.W. Fraser, J.F. Pearson, J.E. Lees and W.B. Feller, “Advances in Microchannel Plate Detectors”, Proc. SPIE Vol. 982 (1988).

2. "High-resolution microchannel plate image tube results", C.B. Johnson, Stanley B. Patton and E.J. Bender, Journal of Electronic Imaging Vol 1(3), pp 252-255 (July 1992).

3. E.H. Eberhart, “An Operational Model for Microchannel Plate Devices”, IEEE Transactions on Nuclear Science Vol 28, pp-712-717, 1980.

4. "Near Earth asteroid rendezvous spacecraft overview", Santo, A.G.; Lee, S.C.; Cheng, A.F., Aerospace Applications Conference, 1996, Proceedings, IEEE Vol. 2, pp. 131 -144, 3-10 Feb. 1996,

5. William L.Wolfe and George J. Zissis, "The Infrared Handbook", Environmental Research Institute of Michigan, 1989.

6. "Validation of Synthetic Imagery for Night Vision Devices", Paul J. Thomas, Robert S. Allison, Sion Jennings, Kevin Yip, Eugene Savchenko, Isaac Tsang, Todd Macuda, Richard Hornsey, SPIE Vol. 5332, pp 1000-1012 (2004).

7. R.D. Burns, J. Shah, Canaan Hong, S. Pepic, Ji Soo Lee, R.I. Hornsey, P. Thomas, “Object location and centroiding techniques with CMOS active pixel sensors", IEEE Transactions on Electron Devices Vol. 50, pp. 2369-2377, 2003.

8. C. Thomas, R. Hornsey, K. Yip, M. Mullins, P. Thomas, CMOS Imager Design for Fast Centroid Readout, IEEE CCECE 2004, Monday May 03, Session \#5, paper \#6.

9. "Modern Theory and Applications of Photocathodes", W.E. Spicer and A. Herrera-Gomez, Proc. SPIE Vol. 2022, pp. 18-32 (1993). 
10. "Brief history of photoemissive materials", Alfred H. Sommer, Proc. SPIE Vol. 2022, pp. 2-17 (1993).

11. W.E. Flynt, "Characterization of Some Common CRT Phosphors", in "Ultrahigh Speed and High-Speed

Photography”, Photonics and Videography '89, G.L. Stradling, editor, Proc SPIE Vol 1155, pp 123-130, 1989.

12. "Scientific Charge-Coupled Devices", James R. Janesick, SPIE Optical Engineering Press, Bellingham, Washington (2001).

13. "A System Engineering Approach to Imaging”, Norman S. Kopeika, SPIE Optical Engineering Press, Bellingham, Washington (1998).

14. “Optical System Design”, Robert E. Fischer and Biljana Tadic-Galeb, McGraw-Hill, New York (2000).

15. "Vision Chips: Implementing Vision Algorithms with Analog VLSI Circuits", Christof Koch and Hua Li, IEEE Computer Society Press, Los Alamitos, CA (1995).

16. "Triangulation range measurement with a CMOS imager", Ed Shen, Anthony P. Badali, Winnifred Wong, Richard I. Hornsey, Paul J. Thomas, Mayes Mullins, SPIE Vol. 5578, paper number C-49, September 2004. 